\title{
Mean Angular Deviation Minimization To Determine Lattice Parameters in Transmission Kikuchi Diffraction
}

Xin Yi Ling ${ }^{1}$, Jared Lodico², B. C. Regan ${ }^{2}$ and Matthew Mecklenburg ${ }^{3}$

${ }^{1}$ Department of Physics and Astronomy, University of California, Los Angeles, California 90095, United States, Los Angeles, California, United States, ${ }^{2}$ Department of Physics and Astronomy, University of California, Los Angeles, California 90095, United States, United States, ${ }^{3}$ Core Center of Excellence in Nano Imaging (CNI), University of Southern California, Los Angeles, California 90089, United States, United States

* Corresponding author: matthew.mecklenburg@usc.edu

Commercial transmission Kikuchi diffraction (henceforth abbreviated EBSD/TKD) software determines crystal orientations by minimizing the mean angular deviation (MAD) between an idealized model of the crystal and the data. The MAD describes the angular deviation between the measured Kikuchi bands in the TKD pattern and the simulated bands, given a reference crystal structure [1]. This algorithm is wellsuited for determining crystal orientations, but it constrains the input lattice parameters to be constant. Lattice parameters can change due to thermal or mechanical stress [2-4], and so they are not necessarily uniform, even in single crystals. Here we present a method that adapts existing commercial EBSD/TKD software for mapping lattice parameters locally within the crystal.

From a thin flake of MoS2 we collect, using a $30 \mathrm{keV}$ accelerating voltage and a $20^{\circ}$ tilt angle from the horizontal, 117 x 156 TKD patterns. With Oxford Instruments' Aztec and AztecICE analysis software, we obtain the mean angular deviation (MAD) value of the TKD pattern at each beam position with respect to a reference crystal structure (Fig. 1, left). The MAD describes the quality of the fit, and smaller MAD values imply crystal structures that are more similar to the reference structure.

In the reference structures we fix the a- and b-axes lattice parameters while varying the c-axis lattice parameter from $11.80 \AA$ to $12.45 \AA$ in steps of $0.05 \AA$. At a given location on the sample, the MAD values generally vary parabolically with changes in the c-axis lattice parameter (Fig. 1, right). The best-fit c-axis lattice parameter corresponds to the minimum of this curve; displaying this best-fit value as a function of real-space position gives a map of the c-axis lattice parameter (Fig. 2, left). This procedure can be repeated for the $\mathrm{a}$ - and $\mathrm{b}$ - lattice parameters. The c-axis best fit lattice constants are distributed in a double Gaussian, with a main peak at $\mathrm{c}=12.14 \pm 0.01 \AA$ and a much smaller secondary peak at $\mathrm{c}=12.11 \pm 0.01 \AA$ (Fig. 2 , right). Features in the lattice parameter map corresponds to those also present in the kernel average misorientation map (Fig. 2 left, inset). These results indicate that TKD patterns can be analyzed to determine not only crystal orientation and phase, but also the crystal's local lattice parameters. 

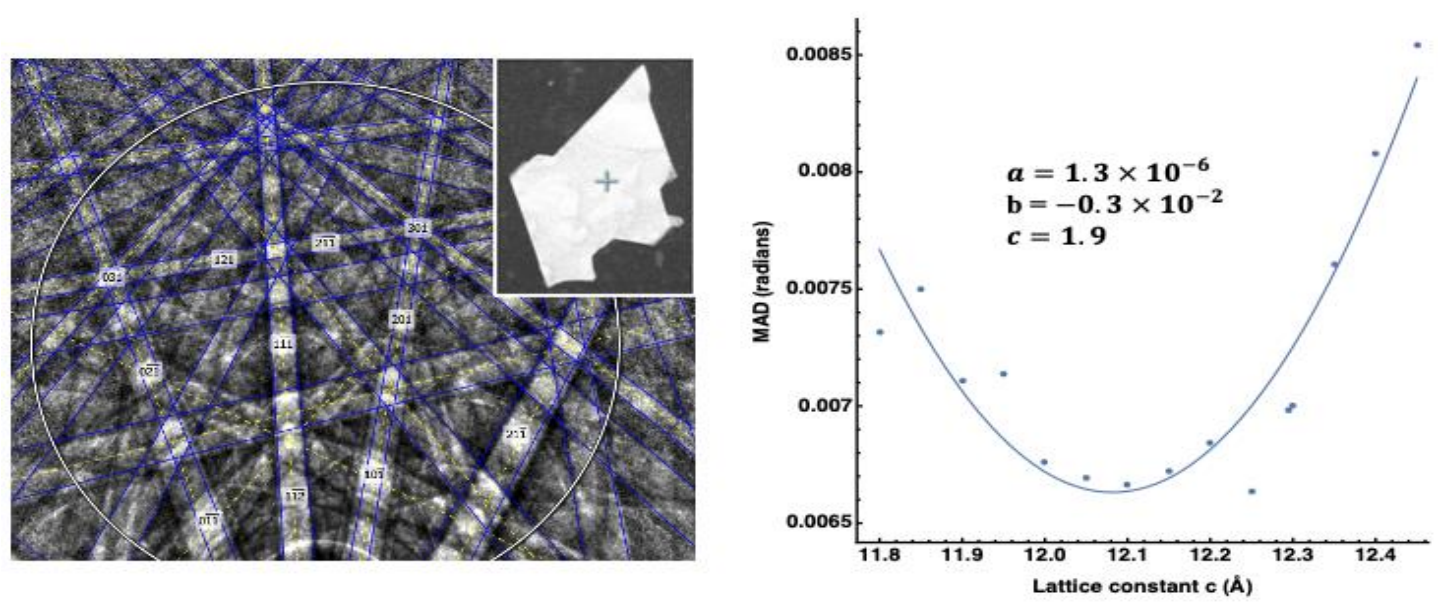

Figure 1. (Left) Typical TKD pattern $(622 \mathrm{px} \times 512 \mathrm{px})$, collected at the location marked by the cross in the inset. The pattern detector was set to solve for 12 bands; blue lines denote detected bands. (Inset) SEM image of sample. (Right) Example of a MAD vs c-axis lattice constant fit located at [50, 72] (origin at top left) on the map. The fit is quadratic: $y=a x^{2}+b x+c$.
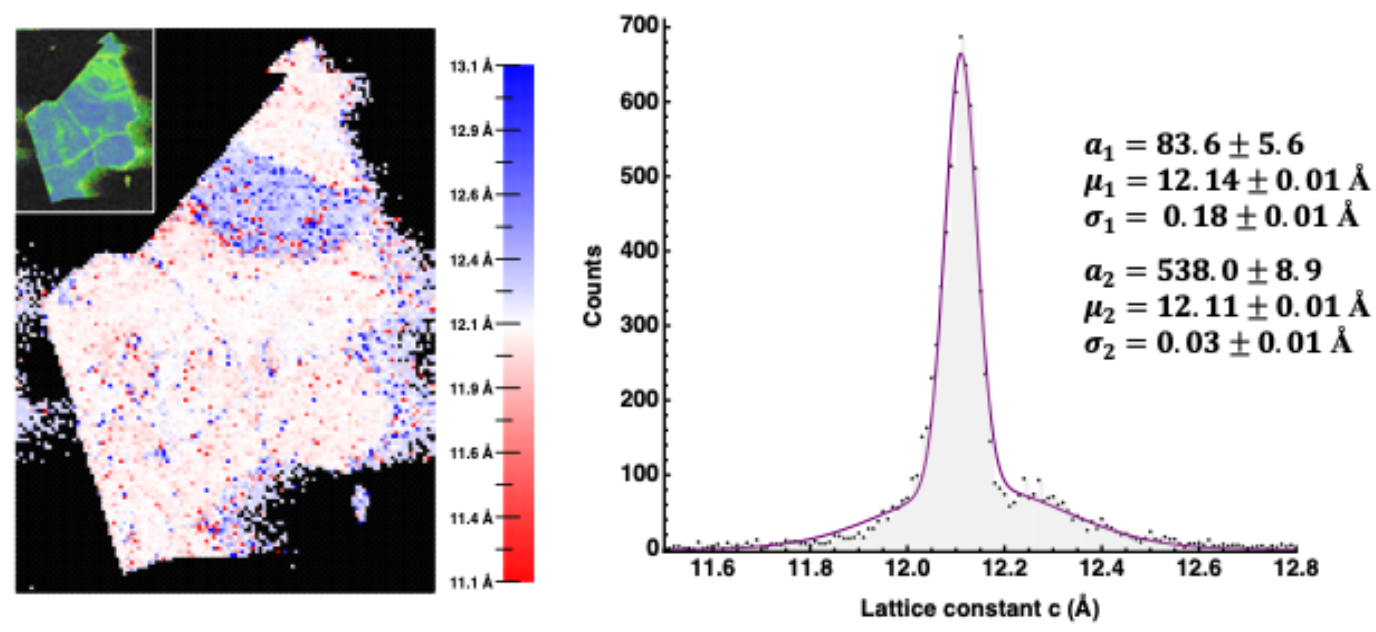

Figure 2. (Left) Map of the best fit c-axis lattice parameter values. (Inset) Kernel average misorientation map of the sample. (Right) Double Gaussian $\left(y=a_{1} \operatorname{Exp}\left[-\left(x-\mu_{1}\right)^{2} /\left(2 \sigma_{1}^{2}\right)\right]+a_{2} \operatorname{Exp}\left[-\left(x-\mu_{2}\right)^{2} /\left(2 \sigma_{2}^{2}\right)\right)\right]$ fit of the optimized c-axis lattice parameters.

\section{References}

[1] Nagaya, T. et al. Minimizing and quantifying mis-indexing in electron backscatter diffraction (EBSD) determinations of antigorite crystal directions. Journal of Structural Geology 95, 127-141 (2017).

[2] Najmaei, S., Liu, Z., Ajayan, P. M. \& Lou, J. Thermal effects on the characteristic Raman spectrum of molybdenum disulfide (MoS2) of varying thicknesses. Appl. Phys. Lett. 100, 013106 (2012).

[3] Yang, L. et al. Lattice strain effects on the optical properties of $\mathrm{MoS}_{2}$ nanosheets. Scientific Reports 4, 5649 (2014).

[4] Hong, J. et al. Exploring atomic defects in molybdenum disulphide monolayers. Nature Communications 6, 6293 (2015).

[5] The data was acquired at the Core Center of Excellence in Nano Imaging (CNI), University of Southern California. This work was supported by National Science Foundation (NSF) Science and Technology Center (STC) award DMR-1548924 (STROBE) and by NSF award DMR-2004897. 